\title{
Targeting for delivery
}

\author{
Translational cancer nanomedicine needs to increasingly exploit newly discovered tumour-targeting strategies as \\ well as the further optimization of proven means to selectively increase the concentration of cytotoxic drugs in \\ solid tumours.
}

S ome working in nanomedicine feel that there is a 'delivery problem'. They mean that delivering drugs to tumours is challenging because of the many pathophysiological barriers (such as the adsorption of protein, sequestration by phagocytic cells and renal clearance) that the nanomedicine needs to navigate before reaching the tumour. The least hopeful also use the phrase to mean that research and development in nanomedicine will not deliver meaningful clinical outcomes. They mention clinical failures of nanoparticlebased anticancer products (in particular the targeted polymeric nanoparticle that precipitated the bankruptcy of BIND therapeutics in 2016), the dearth of nanomedicines available to cancer patients (most notably, that Doxil, a poly(ethylene glycol)-coated liposomal formulation of doxorubicin, and Abraxane, an albuminbound formulation of paclitaxel, were first approved, respectively, in 1995 and 2005), the typically meagre fraction of the injected drug (less than one percent) that reaches the cytosol of tumour cells and, more recently, the announced halting of funding by the United States National Cancer Institute for its Centers of Cancer Nanotechnology Excellence.

However, those who are optimistic about the prospects of cancer nanomedicine allude to the hype cycle, and highlight that reaching the plateau of productivity from a peak of inflated expectations involves enduring a long trough of disillusionment. In fact, last year's approval, by the United States Food and Drug Administration (FDA), of the first nanomedicine based on the mechanism of RNA interference (Patisiran, marketed by Alnylam, a lipidnanoparticle formulation of small interfering RNA for the treatment of familial amyloid polyneuropathy) followed more than a decade of research and development by the company and two decades of preclinical research on 'sequence-based drugs' since RNA interference was discovered in 1998.

The more hopeful also point to the tens of liposomal, polymeric or micellar nanomedicines being tested in advanced clinical trials, and to the many nanopharmaceuticals and nanoparticlebased products currently on the market.

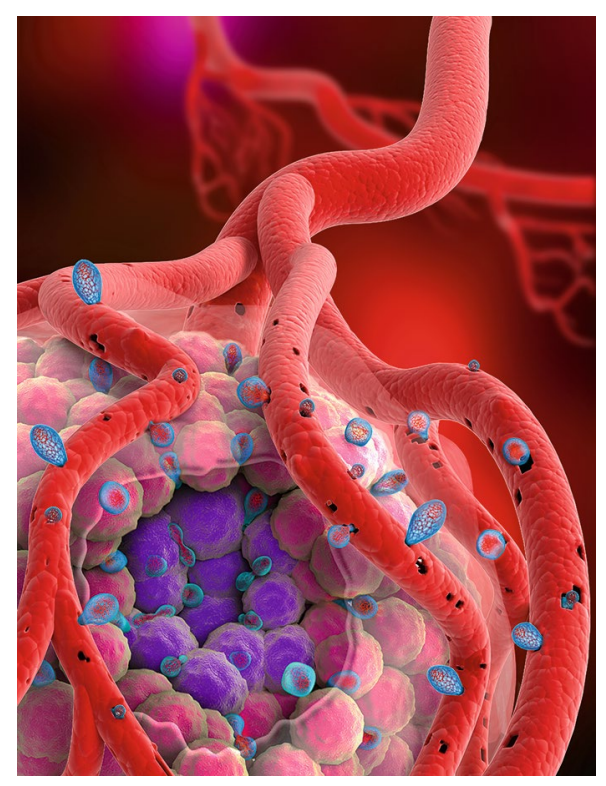

Credit: Huazhong University of Science and Technology

And they are not rattled by the oftenquoted numbers of low delivery efficiency of nanoparticle drugs in tumours; after all, the fraction of the injected dose reaching the tumour does not correlate well with therapeutic outcomes, and is therefore less relevant than classical pharmacological variables (such as the drug's half-life in blood) and the fold-increase in drug concentration in the tumour with respect to the administration of the free drug. Also, for many drugs in human trials, the fraction of the administrated dose that reaches the tumour can be of the order of $10 \%$.

Yet everyone agrees that there are many opportunities. First, patients can be stratified (for example, via magnetic resonance imaging, with contrast enhanced by nanoparticles) according to their tumours' propensity to passively accumulate nanomedicines - the so-called enhanced permeability and retention effect, a term encompassing a range of poorly understood mechanisms. Second, anticancer biologics (such as the monoclonal antibody Herceptin for treating breast cancer) can also benefit from delivery approaches used in nanomedicine. Third, nanomedicines can be made to convert to an active state only via a stimulus present in the tumour's microenvironment, such as its acidic $\mathrm{pH}$; this can make the drug safer elsewhere in the body and allow for bigger doses. Fourth, nanomedicines can be designed to modulate the immune system to recognize cancer cells, or to target and potentiate tumourinfiltrating myeloid cells. Fifth, for some applications, nanomedicines can be designed to be administered locally (via topical or intramuscular routes, for instance), which can drastically reduce off-target effects. Sixth, encapsulation and targeting strategies to maximizing the amount of drug in the tumour can be leveraged in new ways, and also optimized for specific applications.

Three Articles included in this issue exemplify the latter point. In one Article, Xian-Zheng Zhang and colleagues show that phages infecting cancer-promoting bacteria found in tumours in the colon of mice can be used as 'beacons' to augment the intratumoral concentration of nanoparticles made of dextran (a prebiotic polysaccharide) and encapsulating irinotecan - a firstline chemotherapy drug for colorectal cancer. After binding to the phages via cycloaddition, the dextran nanoparticles also promoted the growth of gut bacteria with anticancer properties. The use of phages to increase the concentration of a drug in colonic tumours will inspire new tumourtargeting drug-delivery strategies.

In another Article, Masakazu Kamata and colleagues report that the concentration of intravenously delivered rituximab - the first anticancer antibody approved by the FDA - in metastases of B-cell lymphoma in the brain of humanized mice can be increased tenfold by encapsulating the antibody within a biodegradable crosslinked zwitterionic polymer and by conjugating the polymer with a ligand known to be highly expressed in B-cell lymphoma. The optimization of formulations of encapsulated therapeutic antibodies targeted to metastases could be explored for the treatment of other cancers resistant to first-line therapies.

In a third Article, Lu Gan, Bo Huang and Xiangliang Yang show that mechanically softer drug-carrying vesicles more easily extravasate and penetrate into tumour tissue, and that they are preferentially 
taken up there by highly tumorigenic cells (pictured). They showed this with extracellular vesicles derived from tumourrepopulating cells - a soft tumorigenic subpopulation of cancer cells, isolated from soft gels, that is also highly resistant to chemotherapy drugs - in comparison to microparticles derived from differentiated tumour cells grown on conventional tissueculture plastic. Should microparticle softness boost tumoral accumulation more generally, some of the currently used delivery vehicles might benefit from formulations that make them softer.

Either through the discovery of new mechanisms or via the painstaking optimization of reliable approaches, increasing the concentration of a cytotoxic drug in tumour cells, and directing effector molecules and biologics to immune cells, stromal cells or bacteria in tumours, will remain difficult yet achievable targets in the design of nanoscale and microscale formulations. And regardless of whether they are labelled as 'delivery problems' or 'delivery opportunities', strategies to overcome the biological barriers to drug delivery should be at the forefront of basic and translational nanomedicine.

Published online: 9 September 2019

https://doi.org/10.1038/s41551-019-0457-5 